\title{
Benjamin-Ono-Burgers-MKdV Equation for Algebraic Rossby Solitary Waves in Stratified Fluids and Conservation Laws
}

\author{
Hongwei Yang, ${ }^{1}$ Shanshan Jin, ${ }^{1,2}$ and Baoshu Yin ${ }^{3,4}$ \\ ${ }^{1}$ College of Mathematics and System Science, Shandong University of Science and Technology, Qingdao 266590, China \\ ${ }^{2}$ The First Institute of Oceanography, SOA, Qingdao 266061, China \\ ${ }^{3}$ Institute of Oceanology, China Academy of Sciences, Qingdao 266071, China \\ ${ }^{4}$ Key Laboratory of Ocean Circulation and Wave, Chinese Academy of Sciences, Qingdao 266071, China
}

Correspondence should be addressed to Baoshu Yin; baoshuyin@126.com

Received 2 July 2014; Revised 25 August 2014; Accepted 26 August 2014; Published 16 October 2014

Academic Editor: Yufeng Zhang

Copyright (C) 2014 Hongwei Yang et al. This is an open access article distributed under the Creative Commons Attribution License, which permits unrestricted use, distribution, and reproduction in any medium, provided the original work is properly cited.

In the paper, by using multiple-scale method, the Benjamin-Ono-Burgers-MKdV (BO-B-MKdV) equation is obtained which governs algebraic Rossby solitary waves in stratified fluids. This equation is first derived for Rossby waves. By analysis and calculation, some conservation laws are derived from the BO-B-MKdV equation without dissipation. The results show that the mass, momentum, energy, and velocity of the center of gravity of algebraic Rossby waves are conserved and the presence of a small dissipation destroys these conservations.

\section{Introduction}

Nonlinear waves are crucial for the dynamics of the ocean and atmosphere [1-4]; Rossby waves hold a central position in nonlinear waves. In the past decades, much attention has been paid to the Rossby solitary waves. A number of analytical studies of evolution equations governing such waves have been carried out. According to the governing equation, Rossby solitary waves could roughly be divided into two categories: one is the classical solitary waves and the evolution of Rossby waves obeys the KdV type equation, such as $\mathrm{KdV}$ equation $[5,6]$, MKdV equation $[7,8]$, and Boussinesq equation [9]; the outstanding feature of this type solitary waves is that they are very stable and such a set of solitary waves are called soliton; the other is the algebraic solitary waves and the behavior of Rossby waves is governed by an integrodifferential equation, including Benjamin-Ono (BO) equation [10, 11], Intermediate-LongWave (ILW) equation [12], and Boussinesq-BO equation [13]; furthermore, the waveform of the algebraic solitary waves vanishes algebraically as $|x| \rightarrow \infty$.

The aim of the paper is to derive a new equation which governs the behavior of algebraic Rossby solitary waves. From the quasigeostrophic potential vorticity equation in stratified fluids, a new equation (BO-B-MKdV) is generated and is suitable for describing the evolution of Rossby solitary waves. The BO-B-MKdV equation includes dissipation effect and dispersion effect. It is a meaningful expanding for the results in $[6,10]$. Based on the BO-B-MKdV equation, the conservation laws associated with the equation and dissipation effect are discussed and some conserved quantities of Rossby solitary waves are obtained.

\section{Mathematics Model}

The adiabatic potential vorticity equation is in the following form [14]:

$$
\left(\frac{\partial}{\partial t}+\frac{\partial \Psi}{\partial x} \frac{\partial}{\partial y}-\frac{\partial \Psi}{\partial y} \frac{\partial}{\partial x}\right)\left[\nabla^{2} \Psi+f+\frac{f}{\rho_{s}}\left(\frac{\rho_{s}}{s} \frac{\partial \Psi}{\partial z}\right)\right]=0,
$$

where $\Psi$ is the dimensionless stream function; $s=N^{2} / f$, $N(z)$ is the Brunt-Vaisala frequency and is a measure of stability of the stratification; $f$ is called Coriolis parameter; $\rho_{s}$ is density; and $\nabla^{2}=\partial^{2} / \partial x^{2}+\partial^{2} / \partial y^{2}$ denotes the twodimensional Laplace operator. 
The lower boundary condition can be obtained from the thermal equation including dissipation as follows:

$$
\left(\frac{\partial}{\partial t}+\frac{\partial \Psi}{\partial x} \frac{\partial}{\partial y}-\frac{\partial \Psi}{\partial y} \frac{\partial}{\partial x}\right) \frac{\partial \Psi}{\partial z}+\lambda s\left(\frac{\partial^{2} \Psi}{\partial x^{2}}+\frac{\partial^{2} \Psi}{\partial y^{2}}\right)=0
$$

where $\lambda>0$ is dissipation coefficient.

In order to consider the role of nonlinearity, assume the following shear flows:

$$
U= \begin{cases}u_{1} & y<-L, \\ u(y, z) & -L \geq y \leq L, \\ u_{2} & y>L,\end{cases}
$$

where $u_{1}, u_{2}$ are constants. For simplicity, $u(y)$ is assumed to be smooth across $y=-L$ and $y=L$. In order to consider weakly nonlinear perturbation on a zonal flow, assume

$$
\Psi=-\int^{y}\left(U(s, z)+\varepsilon^{2} \alpha\right) d s+\varepsilon \psi
$$

and then in the domain $[-L, L]$, we take $\lambda=\varepsilon^{3} \lambda_{0}$. Equations (1) and (2) can be written as the following perturbation equations:

$$
\begin{gathered}
{\left[\frac{\partial}{\partial t}+\left(u+\varepsilon^{2} \alpha\right) \frac{\partial}{\partial x}+\varepsilon\left(\frac{\partial \psi}{\partial x} \frac{\partial}{\partial y}-\frac{\partial \psi}{\partial y} \frac{\partial}{\partial x}\right)\right]} \\
\times\left[\nabla^{2} \psi+\frac{f}{\rho_{s}} \frac{\partial}{\partial z}\left(\frac{\rho_{s}}{s} \frac{\partial \psi}{\partial z}\right)\right]+\bar{\beta} \frac{\partial \psi}{\partial x}=0 \\
{\left[\frac{\partial}{\partial t}+\left(u+\varepsilon^{2} \alpha\right) \frac{\partial}{\partial x}+\varepsilon\left(\frac{\partial \psi}{\partial x} \frac{\partial}{\partial y}-\frac{\partial \psi}{\partial y} \frac{\partial}{\partial x}\right)\right]} \\
\times \frac{\partial \psi}{\partial z}-\frac{\partial u}{\partial z} \frac{\partial \psi}{\partial x}+\varepsilon^{3} \lambda_{0} s \nabla^{2} \psi=0 \\
z=0
\end{gathered}
$$

where $\bar{\beta}=\beta-\left(\partial^{2} u / \partial y^{2}\right)-\left(f / \rho_{s}\right)(\partial / \partial z)\left(\left(\rho_{s} / s\right)(\partial u / \partial z)\right)$ and $\beta$ is a constant. The upper boundary condition is as follows:

$$
\rho_{s} \psi \longrightarrow 0, \quad z \longrightarrow \infty \text {. }
$$

In the domains $(-\infty,-L)$ and $(L, \infty)$, the parameter $\beta$ is smaller than that in the domain $[-L, L]$; here we assume $\beta=0$ for $|y|>L$. Then, the governing equations in these areas are

$$
\begin{gathered}
{\left[\frac{\partial}{\partial t}+\left(u_{1,2}+\varepsilon^{2} \alpha\right) \frac{\partial}{\partial x}+\varepsilon\left(\frac{\partial \psi}{\partial x} \frac{\partial}{\partial y}-\frac{\partial \psi}{\partial y} \frac{\partial}{\partial x}\right)\right]} \\
\times\left[\nabla^{2} \psi+\frac{f}{\rho_{s}} \frac{\partial}{\partial z}\left(\frac{\rho_{s}}{s} \frac{\partial \psi}{\partial z}\right)\right]=0, \quad|y|>L .
\end{gathered}
$$

Here, the subscript denotes that for $y<-L$ and for $y>L$, respectively.

In order to achieve a balance between nonlinearity and dispersion, we introduce the following stretching transformation and the perturbation expansion of $\psi_{\text {in }}$ in the domain $[-L, L]$ :

$$
\begin{gathered}
X=\varepsilon x, \quad T=\varepsilon^{3} t, \quad y=y, \quad z=z ; \\
\psi_{\text {in }}=\psi_{1}(X, y, T)+\varepsilon \psi_{2}(X, y, T)+\cdots .
\end{gathered}
$$

Separating $\psi_{1}$ as $\psi_{1}=A(X, T) \phi_{1}(y, z)$, defining the operator $L$ as

$$
L=\frac{\partial^{2}}{\partial y^{2}}+\frac{f}{\rho_{s}} \frac{\partial}{\partial z}\left(\frac{\rho_{s}}{s} \frac{\partial}{\partial z}\right)+\frac{\bar{\beta}}{u}
$$

then substituting (8) into (5), we get $O(\varepsilon)$ :

$$
\begin{gathered}
L\left(\phi_{1}(y, z)\right)=0 ; \\
u \frac{\partial \phi_{1}}{\partial z}-\frac{\partial u}{\partial z} \phi_{1}=0, \quad z=0 ; \\
\rho_{s} \phi_{1} \longrightarrow 0, \quad z \longrightarrow \infty .
\end{gathered}
$$

Equation (10) is an eigenvalue problem and describes the space structure of the wave along direction. $A(X, T)$ is the unknown amplitude in the order $O(\varepsilon)$ and needs to solve higher order equations.

Assuming $\psi_{2}=(1 / 2) A^{2}(X, T) \phi_{2}(y, z)$, proceeding to $O\left(\varepsilon^{2}\right)$, we obtain

$$
\begin{gathered}
L\left(\phi_{2}(y, z)\right)=\left(\frac{\bar{\beta}}{u}\right)_{y} \frac{\phi_{1}^{2}}{u} ; \\
\rho_{s} \phi_{2} \longrightarrow 0, \quad z \longrightarrow \infty \\
u \frac{\partial \phi_{2}}{\partial z}-\frac{\partial u}{\partial z} \phi_{2}=-\left(\phi_{1} \frac{\partial^{2} \phi_{1}}{\partial y \partial z}-\frac{\partial \phi_{1}}{\partial y} \frac{\partial \phi_{1}}{\partial z}\right), \quad z=0 .
\end{gathered}
$$

To $O\left(\varepsilon^{3}\right)$, we have

$$
\begin{aligned}
u L\left(\frac{\partial \psi_{3}}{\partial X}\right)= & \left(\frac{\partial A}{\partial T}+\alpha \frac{\partial A}{\partial X}\right) \frac{\bar{\beta} \phi_{1}}{u} \\
& +A^{2} \frac{\partial A}{\partial X}\left\{\frac{\phi_{1} \phi_{2}}{2 u}\left(\frac{\bar{\beta}}{u}\right)_{y}-\frac{\phi_{1}^{3}}{2}\left[\frac{1}{u}\left(\frac{\bar{\beta}}{u}\right)_{y}\right]_{y}\right\} \\
& -u \phi_{1} \frac{\partial^{3} A}{\partial X^{3}} \\
u \frac{\partial}{\partial z} \frac{\partial \psi_{3}}{\partial X}-\frac{\partial u}{\partial z} \frac{\partial \psi_{3}}{\partial X} & \left(\frac{\partial A}{\partial T}+\alpha \frac{\partial A}{\partial X}\right) \frac{\partial \phi_{1}}{\partial z}-A^{2} \frac{\partial A}{\partial X} \\
= & \left(\frac{\phi_{1}}{2} \frac{\partial^{2} \phi_{2}}{\partial y \partial z}-\frac{\partial \phi_{1}}{\partial y} \frac{\partial \phi_{2}}{\partial z}+\phi_{2} \frac{\partial^{2} \phi_{1}}{\partial y \partial z}\right. \\
& \left.-\frac{1}{2} \frac{\partial \phi_{2}}{\partial y} \frac{\partial \phi_{1}}{\partial z}\right)+A \lambda_{0} s \frac{\partial^{2} \phi_{1}}{\partial y^{2}} \quad z=0 \\
& \rho_{s} \psi_{3} \longrightarrow 0, \quad z
\end{aligned}
$$


Multiplying both the sides of the first equation of (12) by $\rho_{s} \phi_{1} / u$ and integrating it over $y$ and $z$ lead to

$$
\begin{aligned}
\int_{0}^{+\infty} & \left.\frac{\partial}{\partial X} \rho_{s}\left[\phi_{1} \frac{\partial}{\partial y} \psi_{3}-\psi_{3} \frac{\partial}{\partial y} \phi_{1}\right]\right|_{-L} ^{L} d z \\
& +\left.\int_{-L}^{L} \frac{\partial}{\partial X} \frac{f \rho_{s}}{s}\left[\phi_{1} \frac{\partial}{\partial z} \psi_{3}-\psi_{3} \frac{\partial}{\partial z} \phi_{1}\right]\right|_{0} ^{+\infty} d y \\
= & \left.\frac{\partial}{\partial T}+\alpha \frac{\partial}{\partial X}\right) A \int_{-L}^{L} \int_{0}^{+\infty} \rho_{s} \frac{\phi_{1}^{2}}{u^{2}} \bar{\beta} d y d z \\
& -\frac{\partial^{3}}{\partial X^{3}} \int_{-L}^{L} \rho_{s} \phi_{1}^{2} d y d z+A^{2} \frac{\partial A}{\partial X} \\
& \times \int_{-L}^{L} \int_{0}^{+\infty}\left\{\frac{\rho_{s} \phi_{1}^{2} \phi_{2}}{2 u^{2}}\left(\frac{\bar{\beta}}{u}\right)_{y}\right. \\
& \left.-\frac{\rho_{s} \phi_{1}^{4}}{2 u}\left[\frac{1}{u}\left(\frac{\bar{\beta}}{u}\right)_{y}\right]_{y}\right\} d y d z .
\end{aligned}
$$

In (13), if the boundary conditions of $\phi_{1}$ and $\psi_{3}$ are known, the equation governing the amplitude $A$ will be determined. So in the following, we will consider the boundary conditions of $\phi_{1}$ and $\psi_{3}$.

For the two external regions of $|y|>L$, consider the following transformations:

$$
T=\varepsilon^{3} t, \quad \xi=x, \quad y=y, \quad Z=\varepsilon z
$$

and the external stream function $\psi_{\mathrm{ex}}$ is set to

$$
\psi_{\mathrm{ex}}=\tilde{\psi}(\xi, y, Z, T, \varepsilon) .
$$

Substituting (14) and (15) into (7) based on the lowest-order equation of the external region, we can obtain

$$
\left(\frac{\partial^{2}}{\partial \xi^{2}}+\frac{\partial^{2}}{\partial y^{2}}\right) \widetilde{\psi}(\xi, y, Z, T, \varepsilon)=0
$$

The solution of (16) satisfies

$$
\begin{gathered}
\tilde{\psi}=\left.\frac{ \pm P}{\pi} \int_{-\infty}^{+\infty} \widetilde{\psi}\right|_{\substack{\xi=\xi^{\prime} \\
y= \pm L}} \frac{(y \mp L) d \xi^{\prime}}{(y \mp L)^{2}+\left(\xi-\xi^{\prime}\right)^{2}}, \\
\frac{\partial \widetilde{\psi}}{\partial y}=\left.\frac{ \pm P}{\pi} \int_{-\infty}^{+\infty} \widetilde{\psi}\right|_{\substack{\xi=\xi^{\prime} \\
y= \pm L}} \frac{\left[\left(\xi-\xi^{\prime}\right)^{2}-(y \mp L)^{2}\right] d \xi^{\prime}}{\left[(y \mp L)^{2}+\left(\xi-\xi^{\prime}\right)^{2}\right]^{2}},
\end{gathered}
$$

where the upper and the lower signs denote that for $y>L$ and for $y<-L$, respectively, and $P$ stands for the principal value of the integration. Assuming that the inner solution matches smoothly with the outer solutions at $y= \pm L$, then we obtain

$$
\begin{gathered}
\left.\left(\psi_{1}+\varepsilon \psi_{2}+\varepsilon^{2} \psi_{3}\right)\right|_{y= \pm L}=\left.\widetilde{\psi}\right|_{y= \pm L}+O\left(\varepsilon^{3}\right), \\
\left.\frac{\partial\left(\psi_{1}+\varepsilon \psi_{2}+\varepsilon^{2} \psi_{3}\right)}{\partial y}\right|_{y= \pm L}=\left.\frac{\partial \widetilde{\psi}}{\partial y}\right|_{y= \pm L}+O\left(\varepsilon^{3}\right) .
\end{gathered}
$$

Then by employing (18), we get

$$
\begin{gathered}
A \phi_{1}( \pm L)=\left.\widetilde{\psi}\right|_{y= \pm L},\left.\quad \psi_{2}\right|_{y= \pm L}=\left.\psi_{3}\right|_{y= \pm L}=0, \\
\frac{\partial \widetilde{\psi}}{\partial y}=\mp \varepsilon^{2} \phi_{1}( \pm L) \frac{\partial^{2} \mathscr{J}(A(X, T))}{\partial X^{2}}, \\
\left.\frac{\partial \psi_{3}}{\partial y}\right|_{y= \pm L}=\mp \phi_{1}( \pm L) \frac{\partial^{2} \mathscr{J}(A(X, T))}{\partial X^{2}},
\end{gathered}
$$

where $\mathscr{J}(A(X, T)) \equiv(P / \pi) \int_{-\infty}^{+\infty} A\left(X^{\prime}, T\right) \ln \left|X-X^{\prime}\right| d X^{\prime}$. Combining (13) with (19), with the help of (10), (11), and (12), yields

$$
\begin{aligned}
\frac{\partial A}{\partial T}+\alpha \frac{\partial A}{\partial X}+a_{1} A^{2} \frac{\partial A}{\partial X}+a_{2} \frac{\partial^{3} A}{\partial X^{3}}+a_{3} A \\
+a_{4} \frac{\partial^{3}}{\partial X^{3}} \mathscr{J}(A(X, T))=0,
\end{aligned}
$$

and then (20) can be rewritten as follows:

$$
\begin{aligned}
\frac{\partial A}{\partial T} & +\alpha \frac{\partial A}{\partial X}+a_{1} A^{2} \frac{\partial A}{\partial X}+a_{2} \frac{\partial^{3} A}{\partial X^{3}}+a_{3} A \\
& +a_{4} \frac{\partial^{2}}{\partial X^{2}} \mathscr{H}(A(X, T))=0,
\end{aligned}
$$

where

$$
\begin{aligned}
& a=\left\{\int_{-L}^{L}\left[\int_{0}^{+\infty} \frac{\rho_{2} \bar{\beta}}{u^{2}} \phi_{1}^{2} d z-\left.\frac{f \rho_{s}}{s u} \phi_{1} \frac{\partial \phi_{1}}{\partial z}\right|_{z=0}\right] d y\right\}^{-1}, \\
& a_{1}=a \int_{-L}^{L}\left[\int_{0}^{+\infty} \frac{\rho_{s} \phi_{1}^{2} \phi_{2}}{2 u^{2}}\left(\frac{\bar{\beta}}{u}\right)_{y}-\frac{\rho_{s} \phi_{1}^{4}}{2 u}\left(\frac{1}{u}\left(\frac{\bar{\beta}}{u}\right)_{y}\right)_{y} d z\right. \\
& -\frac{f \rho_{s}}{s u} \phi_{1}\left(\frac{\phi_{1}}{2 u} \frac{\partial^{2} \phi_{2}}{\partial y \partial z}-\frac{1}{2 u} \frac{\partial \phi_{2}}{\partial y} \frac{\partial \phi_{1}}{\partial z}\right) \\
& \left.-\frac{1}{u} \frac{\partial \phi_{1}}{\partial y} \frac{\partial \phi_{2}}{\partial z}+\left.\frac{\phi_{2}}{u} \frac{\partial^{2} \phi_{1}}{\partial y \partial z}\right|_{z=0}\right] d y \\
& a_{2}=-a \int_{-L}^{L} \int_{0}^{+\infty} \rho_{s} \phi_{1}^{2} d z d y, \\
& a_{3}=-\left.a \int_{-L}^{L} \frac{\lambda_{0} f \rho_{s} \phi_{1}}{u} \frac{\partial^{2} \phi_{1}}{\partial y^{2}}\right|_{z=0} d y \text {, } \\
& a_{4}=\left.a \int_{0}^{+\infty} \rho_{s} \phi_{1}^{2}\right|_{-L} ^{L} d z \\
& \mathscr{H}(A(X, T)) \equiv \frac{P}{\pi} \int_{-\infty}^{+\infty} \frac{A\left(X^{\prime}, T\right)}{\left(X-X^{\prime}\right)} d X^{\prime}
\end{aligned}
$$

is the well-known Hilbert transform.

Equation (21) is an integrodifferential equation including dissipation effect and dispersion effect. In the absence of 
dissipation effect and $a_{4}=0$, (21) degenerates to the MKdV equation; in the absence of dissipation effect $a_{3} A$ and dispersion effect $A_{X X X}$, (21) degenerates to the generalized $\mathrm{BO}$ equation. Because the term $a_{3} A$ expresses the dissipation effect and has the same physical meaning with the term $\partial^{2} A / \partial X^{2}$ in Burgers equation, so we call (21) BO-B-MKdV equation. As we know that the BO-B-MKdV equation is first obtained here. This equation is greatly different from the common equation to describe algebraic Rossby solitary waves, such as the $\mathrm{BO}$ equation [10] and the BO-Burgers equation; it includes the dissipation effect and dispersion effect and has stronger nonlinearity.

\section{Conservation Laws}

Conservation laws are a common feature of mathematical physics and describe physical properties that remain constant throughout the various processes that occur in the physical world. In physics, "to conserve" something means "to result in no net loss of" that particular component. It is very important in the analysis of unsteady problems of wave propagation. As is known, some famous soliton equations have some conserved quantities. For example, it is also proved that the $\mathrm{BO}$ equation has four conservation laws in [15]. One of the goals of the present paper is to investigate the following questions. Has the BO-B-MKdV equation also conservation laws in the absence of dissipation effect? How to change these conservation quantities in the presence of dissipation effect?

Here, we assume that when $|X| \rightarrow \infty, A, A_{X}, A_{X X}$, and $A_{X X X} \rightarrow 0$. First, (21) can be expressed as follows:

$$
\begin{aligned}
\frac{\partial A}{\partial T}= & -\frac{\partial}{\partial X}\left[\alpha A+\frac{1}{3} a_{1} A^{3}+a_{2} \frac{\partial^{2} A}{\partial X^{2}}+a_{4} \frac{\partial}{\partial X} \mathscr{H}(A(X, T))\right] \\
& -a_{3} A .
\end{aligned}
$$

Integrating (23) with respect to $X$ over $(-\infty,+\infty)$, then we have

$$
C_{1}=\int_{-\infty}^{+\infty} A d X=\exp \left(-a_{3} T\right) \int_{-\infty}^{+\infty} A(X, 0) d X .
$$

From (24), we find that $C_{1}$ decreases exponentially with the increasing of time $T$ and the coefficient $a_{3}$, while $a_{3}$ relates to the dissipation coefficient $\lambda_{0}$. This shows that the dissipation effect causes the mass of solitary waves to decrease exponentially. When the dissipation effect is absent, the mass of the solitary waves is conserved.

In the following, we multiply (21) by $A(X, T)$ and reorganize the terms to obtain

$$
\begin{aligned}
\frac{1}{2} \frac{\partial A^{2}}{\partial T}=-\frac{\partial}{\partial X}[ & \frac{1}{2} \alpha A^{2}+\frac{1}{4} a_{1} A^{4}+a_{2} A \frac{\partial^{2} A}{\partial X^{2}}+\frac{1}{2} A_{X}^{2} \\
& \left.+a_{4} A \frac{\partial}{\partial X} \mathscr{H}(A(X, T))\right]-A_{X} \mathscr{H}\left(A_{X}\right) \\
& -a_{3} A^{2} .
\end{aligned}
$$

Based on the property of the Hilbert operator $\mathscr{H}$ : $\int_{-\infty}^{+\infty} f(X) \mathscr{H}(f(X)) d X=0$, in which $f(X)$ is an arbitrary function vanishing at infinity and carrying on the integration with respect to $X$ over $(-\infty,+\infty)$ leads to

$$
C_{2}=\int_{-\infty}^{+\infty} A^{2} d X=\exp \left(-2 a_{3} T\right) \int_{-\infty}^{+\infty} A^{2}(X, 0) d X .
$$

Equation (26) shows that the momentum of the solitary waves is conserved without dissipation. Because of the dissipation effect, the momentum of the solitary waves also decreases exponentially with the increasing of time $T$ and the dissipative coefficient $\lambda_{0}$. The rate of decline of momentum is faster than the rate of mass.

From (24) and (26), we have obtained the conservation of mass and momentum of the solitary waves, now without regard to the dissipation effect, by adding $\left(A^{2}-\right.$ $\left.\left(a_{4} / a_{1}\right) \mathscr{H}\left(A_{X}\right)\right) \times(21)$ to $(\partial / \partial X)(21) \times\left[A_{X}+\left(a_{4} / a_{1}\right) \mathscr{H}(A)\right]$ and integrating it, by virtue of the relation

$$
\frac{\partial^{2} \mathscr{H}(A)}{\partial X^{2}}=\mathscr{H}\left(\frac{\partial^{2} A}{\partial X^{2}}\right), \quad \int_{-\infty}^{+\infty}(u \mathscr{H} v+v \mathscr{H} u) d X=0,
$$

after tedious calculation, we obtain

$$
\frac{d C_{3}}{d T}=\frac{d}{d T} \int_{-\infty}^{+\infty}\left[\frac{1}{3} A^{3}+\frac{1}{2} A_{X}^{2}+\frac{a_{4}}{a_{1}} \frac{\partial A}{\partial X} \mathscr{H}(A)\right] d X=0 .
$$

$\mathrm{C}_{3}$ is regarded as the energy of the solitary waves. So we can conclude that the energy of the solitary waves is conserved without dissipation.

Finally, let us define a quantity related to the phase of the solitary waves:

$$
\widetilde{C}_{4}=\frac{d}{d T} \int_{-\infty}^{+\infty} X A d X .
$$

Then, employing the momentum of the solitary waves $C_{2}$ is a time-invariant quantity and the above assumptions $A, A_{X}$, $A_{X X}$, and $A_{X X X}$ vanish as $|X| \rightarrow \infty$ as well as $\lambda_{0}=0$; we are easy to deduce $d \widetilde{C}_{4} / d T=0$. We construct the velocity of the center of gravity for the ensemble of such waves $C_{4}=\widetilde{C}_{4} / C_{1}$ [15]. Then, because $C_{1}$ and $\widetilde{C}_{4}$ are time-invariant quantities, we are easy to obtain $d C_{4} / d T=0$; that is, the velocity of the center of gravity is conserved without dissipation.

\section{Conclusions}

In this paper, a new governing equation is derived by the multiple-scale method to describe the amplitude of algebraic Rossby solitary waves in stratified fluids under the influence of dissipation. By analysis and calculation, we obtain four conserved quantities as mass, momentum, energy, and velocity of the center of gravity of algebraic Rossby solitary waves without dissipation and draw the conclusion that the dissipation effect causes the mass, the momentum, the energy, and the velocity of the center of gravity to vary. In fact, after 
the above four conservation laws are given, we can wonder whether there exist other conservation laws and whether there is no limit as KdV equation, which remain to be studied in the future. In addition, we will also explore to study the blocking phenomenon in the ocean and atmosphere by using the mathematical model that we establish in this paper in the future.

\section{Conflict of Interests}

The authors declare that there is no conflict of interests regarding the publication of this paper.

\section{Acknowledgments}

This work was supported by Strategic Pioneering Program of Chinese Academy of Sciences (no. XDA 11020104), Global Change and Air-Sea Interaction (no. GASI-03-01-01-02), National Natural Science Foundation of China through Grants (no. 41376030,61201431), Nature Science Foundation of Shandong Province of China (no. ZR2013AQ017), Science and Technology Plan Project of Qingdao (no. 14-2-4-77-jch), Open Fund of the Key Laboratory of Ocean Circulation and Waves, Chinese Academy of Science (no. KLOCAW1401), Open Fund of the Key Laboratory of Data Analysis and Application, State Oceanic Administration (no. LDAA-201304), and the SDUST Research Fund (no. 2012KYTD105).

\section{References}

[1] S. G. H. Philander, "Forced oceanic waves," Reviews of Geophysics, vol. 16, no. 1, pp. 15-46, 1978.

[2] J. P. Boyd, "Equatorial solitary waves. Part 2: envelope solitons," Journal of Physical Oceanography, vol. 13, no. 3, pp. 428-449, 1983.

[3] Z. H. Xu, B. S. Yin, and Y. J. Hou, "Response of internal solitary waves to tropical storm Washi in the northwestern South China Sea," Annales Geophysicae, vol. 29, no. 11, pp. 2181-2187, 2011.

[4] Z. Xu, B. Yin, Y. Hou, and Y. Xu, "Variability of internal tides and near-inertial waves on the continental slope of the northwestern South China Sea," Journal of Geophysical Research C: Oceans, vol. 118, no. 1, pp. 197-211, 2013.

[5] L. G. Redekopp, "On the theory of solitary Rossby waves," Journal of Fluid Mechanics, vol. 82, no. 4, pp. 725-745, 1977.

[6] L. Meng and K. L. Lv, "Dissipation and algebraic solitary long-waves excited by localized topography," Chinese Journal of Computational Physics, vol. 19, no. 2, pp. 159-167, 2002.

[7] M. Wadati, "The modified Korteweg-de Vries equation," Journal of the Physical Society of Japan, vol. 34, pp. 1289-1296, 1973.

[8] J. Song and L. G. Yang, "Modified KdV equation for solitary Rossby waves with effect in barotropic fluids," Chinese Physics $B$, vol. 7, pp. 2873-2877, 2009.

[9] H. W. Yang, B. S. Yin, and Y. L. Shi, "Forced dissipative Boussinesq equation for solitary waves excited by unstable topography," Nonlinear Dynamics, vol. 70, no. 2, pp. 1389-1396, 2012.

[10] H. Ono, "Algebraic Rossby wave soliton," Journal of the Physical Society of Japan, vol. 50, no. 8, pp. 2757-2761, 1981.
[11] D. H. Luo, "On the Benjamin-Ono equation and its generalization in the atmosphere," Science in China B, vol. 32, no. 10, pp. 1233-1245, 1989.

[12] C. G. Koop and G. Butler, "An investigation of internal solitary waves in a two-fluid system," Journal of Fluid Mechanics, vol. 112, pp. 225-251, 1981.

[13] H. W. Yang, X. R. Wang, and B. S. Yin, "A kind of new algebraic Rossby solitary waves generated by periodic external source," Nonlinear Dynamics, vol. 76, no. 3, pp. 1725-1735, 2014.

[14] J. G. Chraney and D. M. Straus, "Form-drag instability, multiple equilibria and propagating planetary waves in baroclinic, orographically forced, planetary wave systems," Journal of the Atmospheric Sciences, vol. 37, pp. 1157-1176, 1980.

[15] H. Ono, "Algebraic solitary waves in stratified fluids," Journal of the Physical Society of Japan, vol. 39, no. 4, pp. 1082-1091, 1975. 


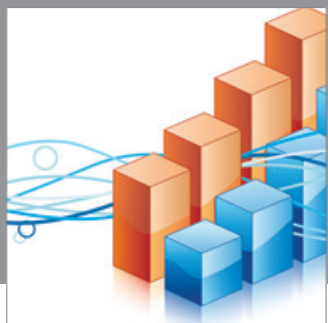

Advances in

Operations Research

mansans

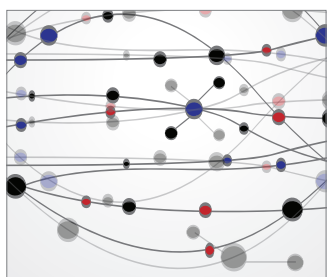

The Scientific World Journal
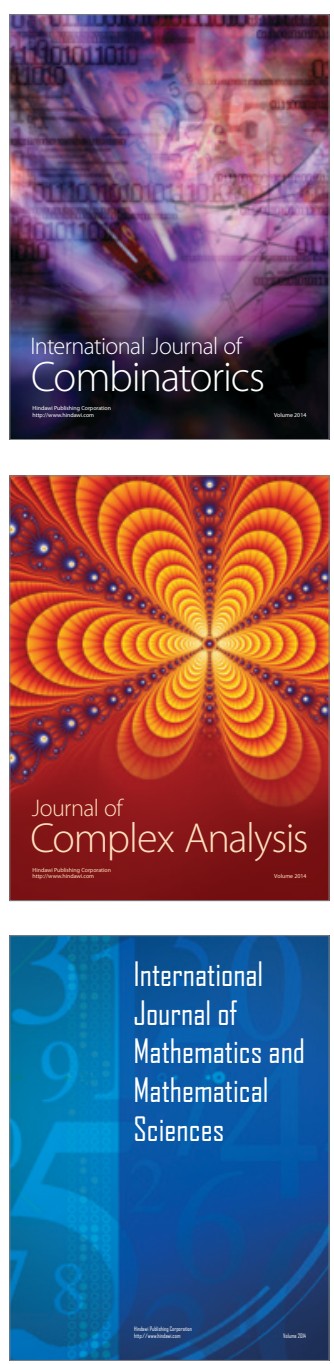
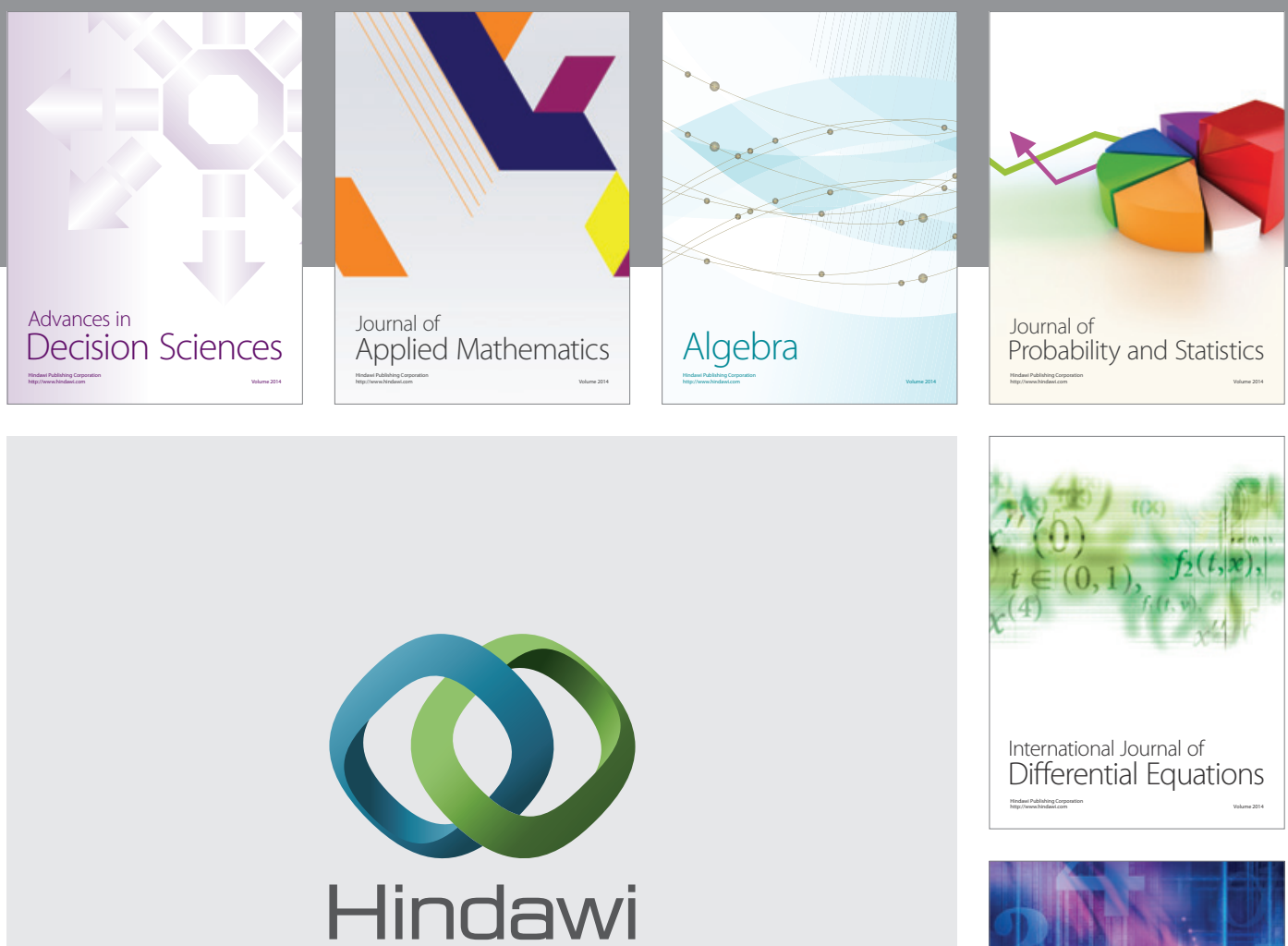

Submit your manuscripts at http://www.hindawi.com
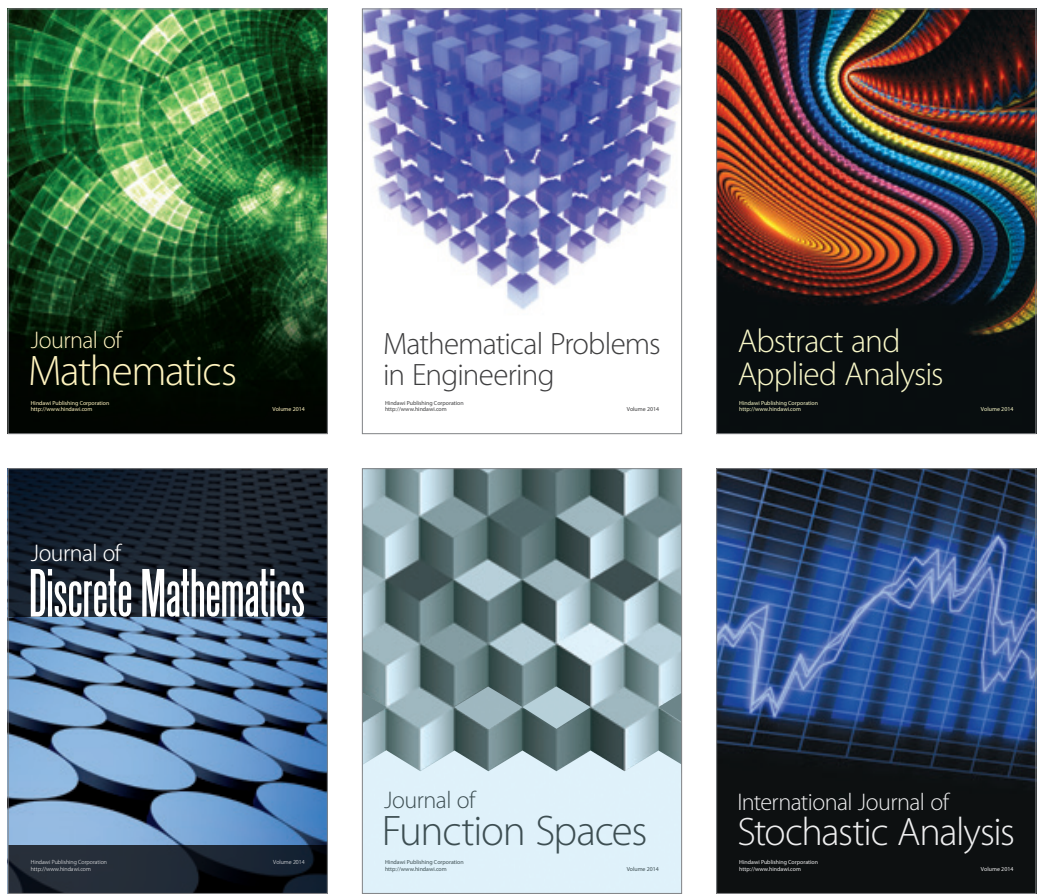

Journal of

Function Spaces

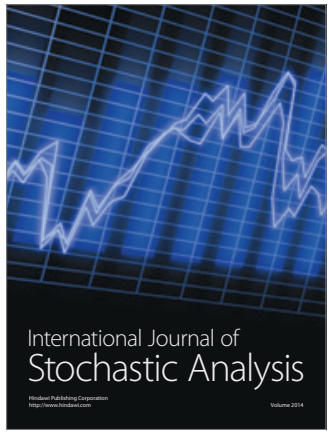

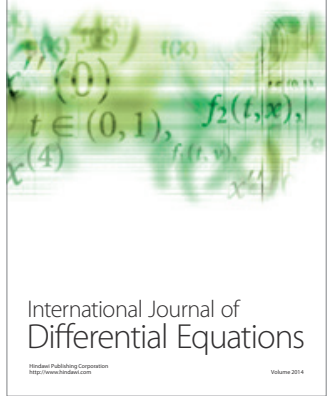
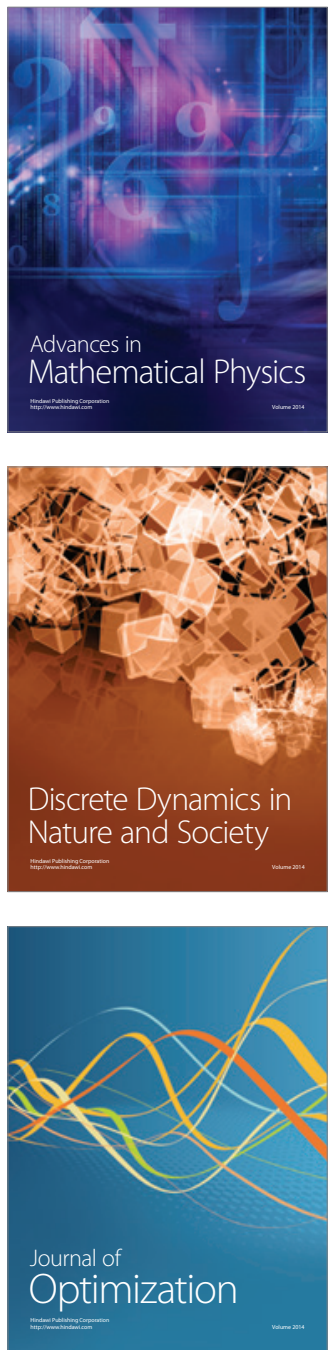